\title{
A Convenient Synthesis of new Phenanthrolinone and Naphthyridinone Derivatives: Evaluation of their Biological activity
}

\author{
Mona A. Hosny ${ }^{a}$, Wafaa A. Mokbel ${ }^{a}$ and Emtithal A. El-Sawi ${ }^{\mathrm{a}}$ \\ ${ }^{a}$ Department of Chemistry, Faculty of Women for Arts Science and Education, \\ Ain Shams University, Cairo, Egypt. \\ E-mail address: monaaminhosny@yahoo.com; wafaamokbel@ymail.com; \\ elsawi_e@yahoo.com
}

\section{Abstract}

A novel and effective synthesis of substituted acetamide via smiles rearrangement is described. Treatment of phenols with 2-chloroacetamide in the presence of sodium hydroxide and DMA where substituted phenols, which contain electron withdrawing groups, are more reactive for smiles rearrangement. The reaction followed by cyclization of the product by E.A.A. afforded the corresponding substituted phenanthrolinone and naphthyridinone in good yields and showed higher activity against $\left(\mathrm{G}^{-}\right.$and $\mathrm{G}^{+}$, Eschierchia coli and Staphylococcus aureus respectively) and good activity toward Aspergillius flavus and Candida albicans.

Keywords: Smiles rearrangement, phenanthrolinone and naphthyridinone.

\section{Council for Innovative Research}

Peer Review Research Publishing System

\section{Journal: Journal of Advances in Chemistry}

Vol. 9, No. 1 


\section{Introduction:}

Smiles rearrangement [1] is one of the most important nucleophilic aromatic substitution reaction which attributed in the recent years in the studying of organic heterocylic compounds and the derivatives of its especially pyridine derivatives [2-7] that has a wide application and more effectiveness in medicinal chemistry and biological activity.

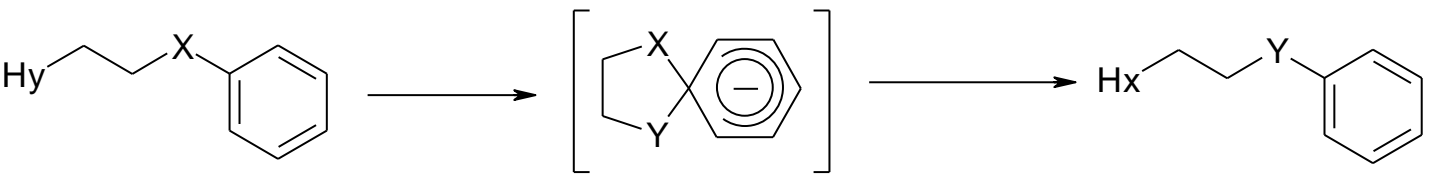

General smiles rearrangement

Scheme (1):

Dihydropyridine drugs, especially nifedipine, nicardipine and amalodipine, shows significant cardiovascular effect in the treatment of hypertension [8]. But, even though these drugs can be utilized, the synthesis of these compounds requires expensive reagents, organic solvents, a long reaction times and the yield result is usually unsatisfactory. Consequently, there has been focused efforts on developing effective and functional method for the implementation of the Hantzsch reaction, and there are potential researches on improved reaction conditions, consuming less reaction times and affording satisfactory yields [9-11].

Heterocyclic compounds exhibited highly biological activity as anti-bacteria, anti-fungel, anti-inflammatory, antipyretic and antihypertensive agents [12-17]. Here, in this work we synthesized phenanthrolinone and napthyridinone derivates by using either phenol or substituted phenol as starting materials which were transformed to amide derivatives and by treatment with E.A.A, the cyclization form took place to afford the substituted pyridinones. This protocol described one pot method proceeds via two-steps process through smiles rearrangement [18] to provide the desired products in good yields as in scheme (2).

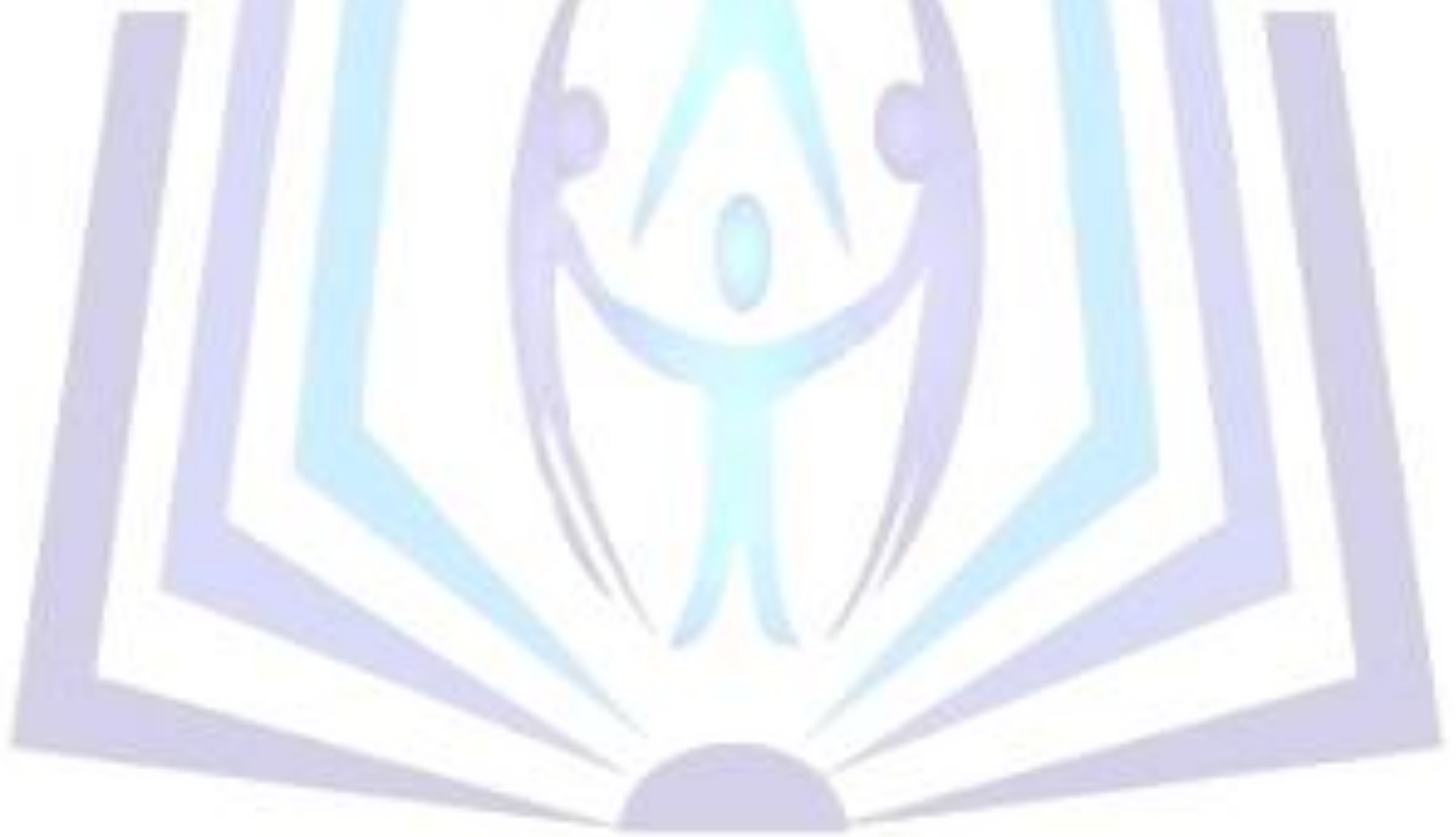


Scheme (2): Alkylation-smiles rearrangement sequence<smiles>CC(C)(Cl)C(N)=O</smiles><smiles>NC(=O)COc1ccccc1</smiles><smiles>CCOC(C)C=NOC</smiles><smiles>O=C(CO)Nc1ccccc1</smiles>

Proposal mechanism for scheme (2)

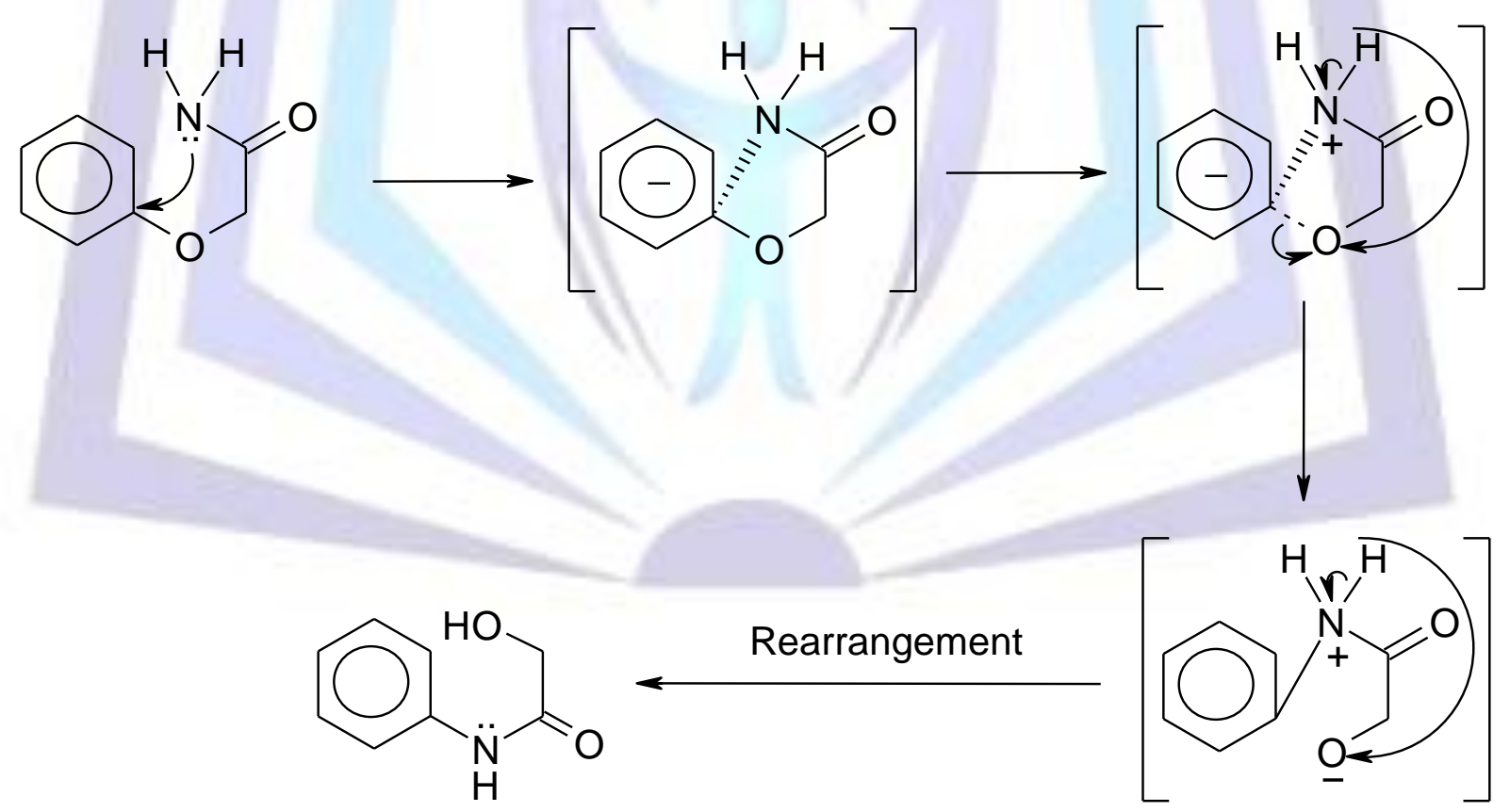




\section{Results and Discussion}

\subsection{Chemistry}

The synthetic route to prepare the different target compounds is described in scheme (3). Compound (1) and (2) afforded (9) and (10) via smiles rearrangement followed by cyclization with ethylacetoacetate to produce phenanthrolinone (11) and naphthyridinone (12) derivatives. Compound (3) and (4) also reacted to produce new compounds (7) and (8) but they failed to follow smiles rearrangement because they react with 2-chloroacetamide to produce the cyclized forms (benzofuranyl and isoquinolinone) (scheme 3) with expected biological activity similar to heterocyclic of medicinal properties and their effect on the blood pressure reduction in spontaneously hypertensive rats [19]; and their cardiotonic and renal vasodilating effects [20].

\section{Scheme (3):}<smiles>Oc1ccc2cccnc2c1</smiles><smiles>Oc1ccncc1</smiles>

$\mathrm{ArOH}$

$3=$<smiles>Oc1ccc(-c2ccc(O)cc2)cc1</smiles><smiles>NC(=O)CCl</smiles>

(in $\mathrm{NaOH}, \mathrm{DMA}$ )<smiles>O=Cc1ccccc1O</smiles><smiles>NC(=O)COc1ccc2cccnc2c1</smiles>

6

$\mathrm{H}_{2} \mathrm{~N}$

$\mathrm{HO}$<smiles>Nc1coc2ccc(-c3ccccc3)cc12</smiles>

7

8

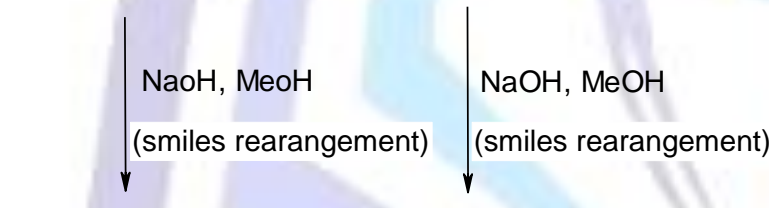<smiles>O=C(CO)Nc1ccc2cccnc2c1</smiles><smiles>O=C(O)CNc1ccncc1</smiles><smiles>CC1=C2C(=O)OCC2NC2c3ncccc3C(CC(F)(F)F)=CC12</smiles><smiles>Cc1c2c(=O)occ-2[nH]c2ccncc12</smiles>

11 


\section{Biological activity:}

Disk diffusion method for filamentous fungi tested by using approved standard method (M38-A) developed by the NCCLS (2002) [21] for evaluating the susceptibilities of filamentous fungi to antifungal agents.

Disk diffusion method for yeasts developed by using approved standard method (M44-P) by the NCCLS (2003)

Standard discs of tetracycline (antibacterial agent), amphotericin B (antifungal agent) served as positive controls for antimicrobial activity but filter discs impregnated with $10 \mu \mathrm{l}$ of solvent (distilled water, chloroform, DMSO) were used as a negative control.

The agar used is Meuller-Hinton agar that is rigorously tested for composition and $\mathrm{pH}$, further the depth of the agar in the plate is a factor to be considered in the disc diffusion method. This method is well documented and standard zones of inhibition have been determined for susceptible and resistant values.

Blank paper disks (Schleicher and Schuell, Spain) with a diameter of $8.0 \mathrm{~mm}$ were impregnated $10 \mu \mathrm{l}$ of tested concentration of the stock solutions. When a filter paper disc impregnated with a tested chemical is placed on agar the chemical will diffuse from the disc into the agar. This diffusion will place the chemical in the agar only around the disc. The solubility of the chemical and its molecular size will determine the size of the area of chemical infiltration around the dis. If an organism is placed on the agar it will not grow in the area around the disc if it is susceptible to the chemical. This area of no growth around the disc is known as a zone of inhibition or "clear zone".

Agar-based methods such as Etest and disk diffusion can be good alternatives because they are simpler and faster than broth-based methods [23, 24].

From the results and regarding that fungi Aspergillus flavus and Candida albicans, most of the previous compounds were found inactive against them except compound (5) which exhibited very higher activity towards them compared with that of standard drugs (tetracycline and amphotericin B), in addition compound (6) which showed higher activity towards Aspergillus flavus only. Also it is interesting to point at that the compounds (9), (11), (12) attributed to good activity against Escherichia coli and staphylococcus aurues.

\begin{tabular}{|c|c|c|c|c|c|}
\hline \multirow{2}{*}{\multicolumn{2}{|c|}{ Compound No. }} & \multicolumn{3}{|c|}{ Inhibition Zone Diameter (mm/mg sample) } & \multirow[b]{2}{*}{$\mathrm{Sa}$} \\
\hline & & Af & $\mathbf{C a}$ & Ec & \\
\hline \multirow{2}{*}{ 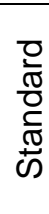 } & $\begin{array}{c}\text { Tetracycline } \\
\text { antibacterial agent }\end{array}$ & & & 33 & 32 \\
\hline & $\begin{array}{l}\text { Amphotericin B } \\
\text { antifungal agent }\end{array}$ & 18 & 20 & - & - \\
\hline & 5 & 30 & 20 & 42 & 47 \\
\hline & 6 & 13 & 0.0 & 0.0 & 0.0 \\
\hline & 9 & 0.0 & 0.0 & 11 & 12 \\
\hline & 11 & 0.0 & 0.0 & 13 & 15 \\
\hline & 12 & 0.0 & 0.0 & 9 & 9 \\
\hline
\end{tabular}

Af - Aspergillus flavus, Ca - Candida albicans, Ec - Escherichia coli, Sa - Staphylococcus aureus

\section{Conclusion:}

In summary we have developed a novel and efficient method for the synthesis of a wide range of phenanthrolinone and naphthyridinone derivatives via the sequential smiles rearrangement followed by intramolecular cyclization in good yields and exhibit antibacterial and antifungal agents. Further work including the application, chemical transformation and biological activity is underway in our laboratory.

\section{Experimental section:}

\subsection{General}

Melting points were taken on Gallen kamp melting point apparatus and were uncorrected. Thin layer chromatography was performed with fluorescent silica gel plates $\mathrm{HF}_{254}$ (Merck), and plates were viewed under $\mathrm{UV}_{254}$ and 265 light. Infrared spectra $\left(\mathrm{v}-\mathrm{cm}^{-1}\right)$ were recorded on Bruker vector Germany and on Mattson FT-IR 1000, using $\mathrm{KBr}$ disks, mass spectra were measured on GCO Finnigan MAT. ${ }^{1} \mathrm{H}-\mathrm{NMR}$ spectra were recorded on Gemini-300 MHZ for ${ }^{1} \mathrm{H}$ and $100 \mathrm{MHz}$ for ${ }^{13} \mathrm{C}$ respectively, in $\mathrm{DMSO}_{-} \mathrm{d}_{6}$ solution and TMS as internal standard in microanalytical center Cairo University - Egypt. Different phenols, 
ethylacetoacetate and DMA were obtained from Fluka or Aldrich. The antibacterial activity was determined in microanalytical center Cairo University - Egypt.

\subsection{General procedure for compounds $5,6,9,10,11,12$}

The phenol (1) or (2) $(1 \mathrm{mmol}),(1 \mathrm{mmol})$ of 2-chloroacetamide and (1 mmol) of sodium hydroxide in DMA (20 ml) were refluxed with stirring for $2 \mathrm{hrs}$ to produce 2 -aryloxyacetamide (5) or (6). Sodium hydroxide (2 mmol) was added to this solution and then the mixture was stirred at $50^{\circ} \mathrm{C}$ to produce $\mathrm{N}$-aryl-2-hydroxy acetamide (9) or (10) which crystallized for each from ethyl alcohol and followed cyclized under the effect of ethylacetoacetate $(1 \mathrm{mmol})$ to produce the compounds (11) or (12) respectively which showed highest inhibition zone against Escherichia coli and staphylococcus aureus, then crystallized from ethyl alcohol.

5.2.1. Compound 5: brown ppt (yield: $56 \%$ m.p. $262^{\circ} \mathrm{C}$. IR $\quad(\mathrm{KBr}) \quad\left(\mathrm{cm}^{-1}\right)$ v:, $3352,3185\left(\mathrm{NH}_{2}\right), 3075$ ( $\mathrm{CH}$, ar.), $2950\left(\mathrm{CH}_{2}\right.$ aliphatic), $1676\left(\mathrm{CO}-\mathrm{NH}_{2}\right), 1559(\mathrm{C}=\mathrm{N}) ; \mathrm{MS}(\mathrm{m} / \mathrm{z} \%): 202(10.46 \%), 145(100 \%)$ ${ }^{1} \mathrm{H}-\mathrm{NMR},\left(\mathrm{DMSO}-\mathrm{d}_{6}\right) \delta: 4.32\left(2 \mathrm{H}, \mathrm{CH}_{2}\right), 7.09\left(2 \mathrm{H}, \mathrm{NH}_{2}\right), 7.41-8.50(5 \mathrm{H}$, ar. protons), 8.81 ( $\mathrm{CH}$, quinoline).

5.2.2. Compound 6: white ppt (yield: $79 \%)$ m.p. $240^{\circ} \mathrm{C}$. IR $\quad(\mathrm{KBr}) \quad\left(\mathrm{cm}^{-1}\right)$ : v: 3389-3220 $\left(\mathrm{NH}_{2}\right), 3060\left(\mathrm{CH}\right.$, ar.), $2928\left(\mathrm{CH}_{2}\right.$ aliphatic), $1648\left(\mathrm{CO}-\mathrm{NH}_{2}\right), 1560(\mathrm{C}=\mathrm{N}) ; \mathrm{MS}(\mathrm{m} / \mathrm{z} \%): 152(54.67 \%), 108$ (100\%); ${ }^{1} \mathrm{H}-\mathrm{NMR},\left(\mathrm{DMSO}-\mathrm{d}_{6}\right) \delta: 4.63\left(2 \mathrm{H}, \mathrm{CH}_{2}\right), 6.05\left(2 \mathrm{H}, \mathrm{NH}_{2}\right), 7.29-7.74(3 \mathrm{H}$, ar. protons), $8.59(\mathrm{CH}$, pyridine).

5.2.3. Compound 9: beige ppt (yield: $49 \%)$ m.p. $340^{\circ} \mathrm{C} . \quad \mathrm{IR} \quad(\mathrm{KBr}) \quad\left(\mathrm{cm}^{-1}\right)$ : v: $3424(\mathrm{OH}), 3185(\mathrm{NH}), 3070\left(\mathrm{CH}\right.$, ar.), $2951\left(\mathrm{CH}_{2}\right.$, aliphatic), $1666(\mathrm{C}=\mathrm{O}), 1628(\mathrm{C}=\mathrm{N}), \mathrm{MS}(\mathrm{m} / \mathrm{z} \%): 202(81.48 \%), 203$ $(\mathrm{M}+1,71.60 \%), 204(\mathrm{M}+2,74.07 \%), 54(100 \%),{ }^{1} \mathrm{H}, \mathrm{NMR},\left(\mathrm{DMSO}-\mathrm{d}_{6}\right) \delta: 1.96(1 \mathrm{H}, \mathrm{OH}), 4.69(2 \mathrm{H}$, metheline), $7.18(1 \mathrm{H}$, $\mathrm{NH}), 7.52-8.54(5 \mathrm{H}$, ar. protons), $8.82(\mathrm{CH}$, quninoline).

5.2.4. Compound 10: beige ppt (yield: 87\%) m.p. decompose $260^{\circ} \mathrm{C}$ IR $(\mathrm{KBr})\left(\mathrm{cm}^{-1}\right)$ : v:3347 (OH), $3285(\mathrm{NH}), 3070\left(\mathrm{CH}\right.$, ar.), $2960\left(\mathrm{CH}_{2}\right.$, aliphatic), $1654(\mathrm{C}=\mathrm{O}) 1556(\mathrm{C}=\mathrm{N}) ; \mathrm{MS}(\mathrm{m} / \mathrm{z} \%): 152$ (65.82\%), 153 (M+1, 79.75\%), 100 (100\%); ${ }^{1} \mathrm{H}-\mathrm{NMR}$ (DMSO-d $)$ ): 1.24(H, OH), $4.52\left(2 \mathrm{H}^{\left.-\mathrm{CH}_{2}\right),} 6.05(1 \mathrm{H}, \mathrm{NH}), 7.42-7.48(3 \mathrm{H}\right.$, Ar.), 7.51 ( $\mathrm{CH}$, pyridine)

5.2.5. Compound 11: deep brown ppt (yield: 52\%) m.p. $150^{\circ} \mathrm{C}$. IR (KBr) (cm $\left.{ }^{-1}\right)$ : v: 3435 (NHstr.), $2981(\mathrm{CH}$, ar.), $2935(\mathrm{CH}$, $\left.\mathrm{CH}_{3}\right), 1725(\mathrm{C}=\mathrm{O}), 1641$ (NH, bend.) $1590(\mathrm{C}=\mathrm{N}), 1409\left(\mathrm{CH}\right.$ bend); MS (m/z\%), $250(52 \%), 124(100 \%) ;{ }^{1}-\mathrm{H}-\mathrm{NMR}$, (DMSO-d6) $\delta: 1.72\left(3 \mathrm{H}, \mathrm{CH}_{3}\right), 4.03(1 \mathrm{H}, \mathrm{NH}), 6.25(1 \mathrm{H},=\mathrm{CH}-\mathrm{O}), 7.25-7.36(4 \mathrm{H}$, ar. $), 7.98\left(\mathrm{CH}\right.$, quninoline); ${ }^{13} \mathrm{C} \mathrm{NMR}(100$ MHZ, DMSO): $\delta 13.9,100.4,107.7,110.1,110.2,110.4,110.5,124.5,144.3,158.6,160.2,168.8,168.9,170.7,197.4$.

5.2.6. Compound 12: deep brown ppt (yield: $82 \%)$ m.p. $140^{\circ} \mathrm{C}$. IR $(\mathrm{KBr})\left(\mathrm{cm}^{-1}\right)$ : v: $3427(\mathrm{NH}), 2980(\mathrm{CH}$, ar.), $2932(\mathrm{CH}$, $\mathrm{CH}_{3}$ ), $1725(\mathrm{C}=\mathrm{O}), 1641$ ( $\mathrm{NH}$ bend.), $1567(\mathrm{C}=\mathrm{N}), 1431\left(\mathrm{CH}\right.$, bend.); MS (m/z\%): 200 (30.22\%), $108(100 \%) ;{ }^{1} \mathrm{H}-\mathrm{NMR}$

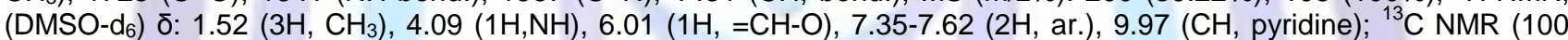
MHZ, DMSO): $\delta 17.7,70.4,71.1,110.7,123.5,123.7,123.9,187.5,188.6,190.9,208.7$.

\subsection{General procedure for compounds 7,8}

The phenol (3) or (4) $(1 \mathrm{mmol}),(1 \mathrm{mmol})$ of 2 -chloroactamide and $(1 \mathrm{mmol})$ of sodium hydroxide in DMA (20 ml) were stirring for $2 \mathrm{hr}$ to produce 4- (3-amino-1-benzofuran-5-yl) phenol (7) or 4-(chloroisoquinolin-3-(4H)-one) (8) which crystallized for each from ethyl alcohol.

5.3.1. Compound 7: white ppt (yield: $88 \%$ m.p. $305^{\circ} \mathrm{C} . \quad \mathrm{IR} \quad(\mathrm{KBr}) \quad\left(\mathrm{cm}^{-1}\right)$ : v: $3599(\mathrm{OH}), 3465,3417\left(\mathrm{NH}_{2}\right), 3276-3168\left(\mathrm{CH}\right.$, ar.), 1599 (NH bend.); $\mathrm{MS}(\mathrm{m} / \mathrm{z} \%): 225(6.38 \%), 185(100 \%) ;{ }^{1} \mathrm{H}-\mathrm{NMR}$,

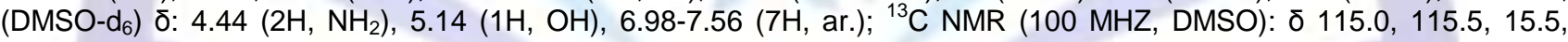
$115.6,117.8,126.8,127.1,127.1,127.2,132.7,156.9,156.9,167.8,169.8$.

5.3.2. Compound 8: brown ppt (yield: $95 \%)$ m.p. above $350^{\circ} \mathrm{C} \mathrm{IR}(\mathrm{KBr})\left(\mathrm{cm}^{-1}\right)$ : v: $3415(\mathrm{OH}$ due to keto-enol form), 2927 $\left(\mathrm{CH}\right.$, ar.), $1675(\mathrm{C}=\mathrm{O}), 1485(\mathrm{C}=\mathrm{N}) ; \mathrm{MS}(\mathrm{m} / \mathrm{z} \%): 179(2.69 \%), 121(100 \%) ;{ }^{1} \mathrm{H}-\mathrm{NMR},\left(\mathrm{DMSO}-\mathrm{d}_{6}\right) \delta: 5.41(1 \mathrm{H},-\mathrm{CH}-\mathrm{Cl})$, 7.20-7.74 (4H, ar.); 8.19 (1H, pyridine); ${ }^{13} \mathrm{C}$ NMR (100 MHZ, DMSO): $\delta 61.2,91.7,113.7,121.2,127.8,127.8,130.7$, 164.6, 189.8 .

\section{References}

[1] Levy, A.A., Rains, H.C., Smiles, S.J., 1931. Chem. Soc. 3264-3269.

[2] Soukri, M., Lazar, S., Akssira, M., Guilaumet, G., 2000. Org. Lett 2, 1557-1560.

[3] Bonini, C., Funicello, M., Scialpi, R., Spagnolo, P., 2003. Tetrahedron 59, 7515-7520.

[4] Tyvorskii, V.I., Bobrov, D.N., Kullnkovich, O.G., Tehrani, K.A., Kimpe, N.D., 2001. Tetrahedron 57, 2051-2055.

[5] Yamamoto, T., Niwa, S., Ohno, S., Ohishi, T., Matsueda, H., Koganei, H., Uneyama, H., Fujita, S., Takeda, T., Kito, M., et al. 2006. Bioorg. Med. Chem. Lett. 16, 798-805.

[6] Rodriguez, H., Martin, O., Ochoa, E., Sunrez, M., Reyes, O., Garay, H., Albericio, F., Martin, N., 2006. QSAR Comb. Sci. 25, 921-927. 
[7] Alvarez, A., Suarez, M., Verdecin, Y., Ochoa, E., Barrie, B., Perez, R., Diaz, M., Martinez-Alvarez, R., Molero, D., Seoane, C., 2006. Heterocycles 68, 1631-1649.

[8] Nekooeian, A.A., Khalili, A., Javidnia, K., Mehdipour, A.R., Miri, R., 2009. Iran. J. Pharmaceut. Res. 8, 193-199.

[9] Polshettiwar, V., Varma, R.S., 2010. RSC Green Chem. Ser. 7, 91-122.

[10] Sivamurugan, V., Vinu, A., Palanichamy, M., Murugesan, V., 2006. Heteroatom. Chem. 17, 267-271.

[11] Narsaiah, A., Venkat, N.B., 2010. Asian J. Chem. 22, 8099-8106.

[12] Bagueley, C.B., Denny, W.A., Atwell, G.A., Finlay, G.J., Rewcastle, G.W., Twigden S.J., Wilson W.R., 1984. Cancer Res. 44, 3245-3251.

[13] Hawtin, R.E., Stockett, D.E., Byl, J.A.W., McDowell, R.S., Tan, N., Arkin, M.R., Conroy, A., Yang, W.N., Osheroff, J.A., 2010. Fox PLOS One 5, e10186.

[14] Kiselev, E., Dexheimer, T.S., Pommier, Y., Cushman, M., 2010. J. Med. Chem. 53, 8716-8726.

[15] El-Subbagh, H.I., Abadi, A.H., Al-Khawad, I.E., Al-Rasshood, A., 1999. Arch. Pharm. Pharm. Med. Chem. $332,19-24$.

[16] Chou, L.C., Tsai, M.T., Hsu, M.H., Wang, S.H., Way, T.D., Huang, C.H., Lin, H.Y., Qian, K., Dong, Y., Lee, K.H., Huang, L.J., Kuo, S.C., 2010. J. Med. Chem. 53, 8047-8058.

[17] You, Q.D., Li, Z.Y., Huang, C.H., Yang, Q., Wang, X.J., Guo, Q.L., 2009. Chen, X.G., He, X.G., Li, T.K., Chern, J.W., J. Med. Chem. 52, 5649-5661.

[18] Masahiro, M., Mitsuhisa, Y., 2005, Organic letters, 7(17) 3629-3631.

[19] Ginn, J.D., Bosanac, T., Chen, R., Cywin, C., Hickey, E., Kashem, M., Kerr, S., Kugler, S., Li, X., Prokopowicz, A., Schlyer, S., Smith, J.D., Turner, M.R., Wu, F., Young, E.R.R., 2010. Bioorganic and Medicinal Chemistry Letters 20, 5153-5156.

[20] Mendez, L.J., Canepa, A.S., Gonzalez, M.G., Bravo, R.D., 2012. Tetrahedron Letters 53, 688-690.

[21] National Committee for Clinical Laboratory Standards (NCCLS). 2002. Reference method for broth dilution antifungal susceptibility testing of conidium-forming filamentous fungi-Proposed standard M38-A. NCCLS, Wayne, PA, USA.

[22] National Committee for Clinical Laboratory standards. 2003. Method for antifungal disk diffusion susceptibility testing of Years: Proposed guideline M44-P. NCCLS, Wayne, PA, USA.

[23] Liebowitz, L.D., Ashbee, H. R., Evans, E.G.V., Chong Y., Mallatova, N., Zaidi, M., Gibbs, D., and Global Antifungal surveillance Group. 2001. Diagn. Diagn. Microbiol. Infect. Disk. 4, 27-33.

[24] Matar, M.J., Ostrosky-Zeichner, L., Paetznick, V.L., Rodriguez, J.R., Chen, E. and Rex, J.H. 2003. Antimicrob. Agents Chemother. 2003, 47, 1647-1651. 